\title{
IMAGO Research Group
}

\author{
Luciano Silva, Olga Regina Pereira Bellon, Maurício Pamplona Segundo, Caroline Mazetto Mendes, \\ Rubisley de Paula Lemes, and Beatriz Trinchão Andrade
}

\begin{abstract}
The IMAGO Research Group was founded in 1996 and accumulated great expertise in range image analysis during its existence. The group has been working on projects related to computer vision, image processing and computer graphics in order to produce solutions for different areas, such as culture, education, social inclusion, health and security. The efforts of the group are currently focused on digital preservation and biometric recognition, in which there are three main ongoing projects: 3D Virtual Museum, 3D Face Recognition and Newborn Identification. These projects have virtual reality and 3D interaction features beyond the HCI requirements, that provides motivation in the use of our systems.
\end{abstract}

Index Terms-Computer vision, image processing, computer graphics, virtual musem, biometrics, medical images.

\section{INTRODUCTION}

The IMAGO Research Group ${ }^{1}$ is part of the Department of Informatics of the Universidade Federal do Paraná (UFPR), Brazil, and was founded by Prof. Olga Regina Pereira Bellon in 1996. Since then, we have been working on projects related to computer vision, image processing and computer graphics, such as digital preservation of cultural and natural assets [1][6], virtual museum [7], face biometrics [8]-[18], newborn identification [19], [20], medical images [21]-[23] and accessibility tools [24]. Several works were published by the IMAGO Research Group in conferences and periodicals and were also acknowledged in undergraduate contests (CTIC/SBC), graduate contests (CTD/SBC) and field-related conferences (SIBGRAPI). Although our main focus is graphics processing, our projects are intrinsically related to virtual reality and $3 \mathrm{D}$ interaction, since the motivation and usability are essential for effective use of the systems we develop.

Our research projects are being developed in collaboration with other organizations, such as the United Nations Educational, Scientific and Cultural Organization (UNESCO), the National Historical and Artistic Heritage Institute (IPHAN), the UFPR Museum of Archeology and Ethnology (MAE) and the UFPR University Hospital. These projects have supported the objectives of our group, which are: (1) to instruct undergraduate and graduate students in research and development activities; (2) to transfer scientific and technologic knowledge; and (3) to develop software applied to culture, education, social inclusion, health and security. Visit the IMAGO Research Group website ${ }^{2}$ for further information.

\section{ONGOING PRojects}

The IMAGO Research Group has a great expertise in range image analysis [25]-[27], and has worked intensively

\footnotetext{
${ }^{1}$ Centro Politécnico, Bairro Jardim das Américas, Curitiba/PR, Brazil, P.O.Box 19092, ZIP Code 81531-980

${ }^{2}$ www.imago.ufpr.br
}

on digital preservation and biometrics in the last years. The three main ongoing projects are: (1) 3D Virtual Museum; (2) 3D Face Recognition; and (3) FootScanAge and FootScanId. A brief history of each project is given below.

\section{A. Digital Preservation and 3D Virtual Museum}

The works on digital preservation began in 2002 with the development of a method to combine 3D images of archaeological objects [1], [2]. Subsequently, a 3D reconstruction system was developed, as well as a 3D Virtual Museum, aiming to digitally preserve and virtually exhibit cultural and natural collections.

The 3D reconstruction system consists of three steps: data acquisition, geometry reconstruction and generation of high resolution textures. In the acquisition step, a laser scanner (Minolta Vivid 910) captures range and low resolution color images and a digital camera (Canon EOS5D) captures high resolution color images.

The geometry reconstruction is subdivided into four main stages: (1) registration, (2) integration, (3) hole filling, and (4) mesh generation [3], [4]. First, multiple views of a object are aligned two by two in the registration stage and after they are all integrated in order to build a single triangle mesh. The holes caused by occlusions and missing data are filled and then we generate a complete $3 \mathrm{D}$ mesh. One of the system features is the use of graphical interfaces that allows the user to control these stages and interact with the 3D model while it is being generated (Figure 1). Finally, the high resolution images are aligned, merged and projected in the 3D model geometry to produce a quality texture for the final 3D model [6].

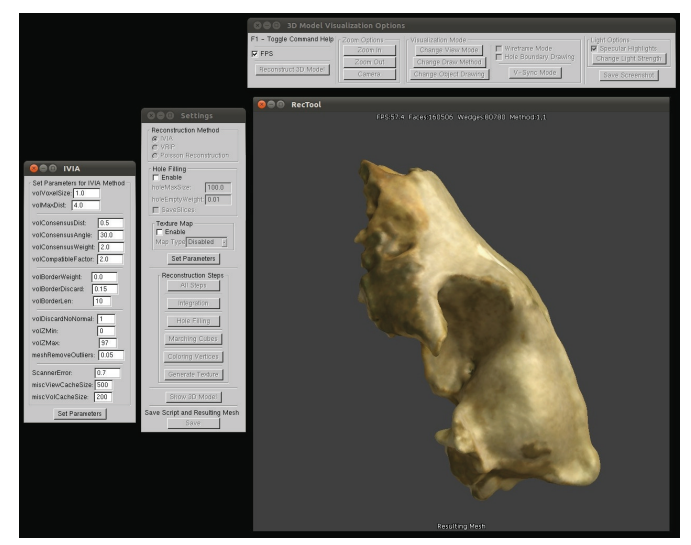

Fig. 1. Interface of the 3D reconstruction system. It has windows with options for the integration stage, model reconstruction and 3D model visualization.

The generated 3D models are displayed in our 3D Virtual Museum [7]. We developed a web-based 3D visualization 
system that allows a fast and practical access to 3D models. The users may interact with the 3D models and access information, images and videos of the preserved objects in the virtual museum website. It is necessary to register to login in the system, and the visualization of the 3D models is limited according to the user access level. Visitors have limited access, but teachers or researchers may request less restricted access in order to visualize 3D models with higher resolution.

Due to our multi-platform 3D visualization tool for web browsers, the user can interact with $3 \mathrm{D}$ models using their preferred web browsers, such as Internet Explorer, Mozilla Firefox, among others. The 3D visualization tool has a simple and intuitive interface which can be customized according to the target group. This is done by editing a configuration file, where the programmer can choose the operations available to manipulate 3D models in the interface, and can choose to use a menu bar and/or the toolbar and the interaction mode (mouse and/or shortcut keys). The interface default image icons can be replaced for drawings or symbols that the programmer considers more intuitive for his/her application users.

Our attention is focused in beginner users, that do not understand some symbols present in the virtual reality interfaces. Figure 2 shows the interface of the 3D visualization tool. The users can perform basic operations such as rotating, moving, zooming, showing the 3D model in wireframe mode, with or without texture, moving the light source and others. To provide an intuitive and simple interface makes the 3D visualization experience more enjoyable and attractive for educational activities.

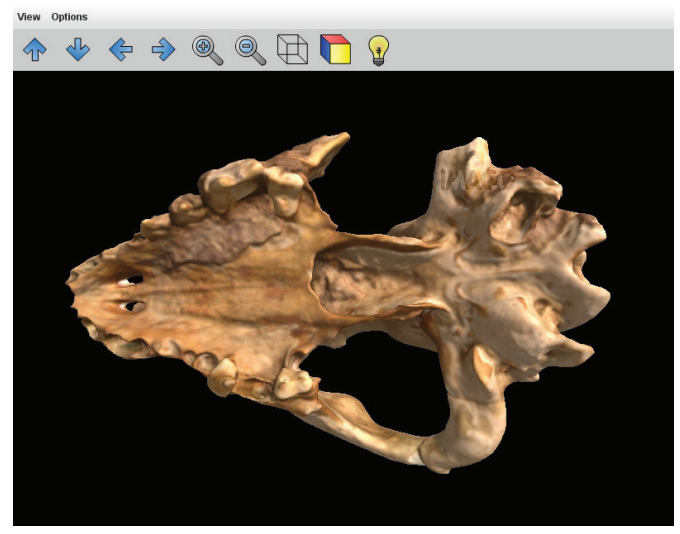

Fig. 2. Visualization of a Protocyon fossil. The Protocyon is an animal that inhabited caves in Brazil during the Pleistocene (period between 1.816.000 and 11.500 years ago). This fossil is property of the UFPR Natural Science Museum.

This project was used on the digital preservation of cultural and scientific heritage from different institutions, such as the Curitiba Metropolitan Art Museum, the UFPR Natural Science Museum (Figure 2) and UFPR MAE (Figure 3). The group is now working in the preservation of baroque sculptures made by Antonio Francisco Lisboa, known as "O Aleijadinho" [5], in collaboration with UNESCO. The sculptures represent twelve biblical prophets and are located in the Bom Jesus de Matosinhos Sanctuary in Congonhas, Brazil. This sanctuary is in the UNESCO World Heritage List, and is composed of a church, a parvis with the prophet sculptures and six stations with scenes from the life and death of Jesus Christ. The main goal of this project is to obtain a register of the sculptures' current state, which may be used in comparative studies and to assist restorations. Figure 4 shows two prophets that were preserved in this collaboration.

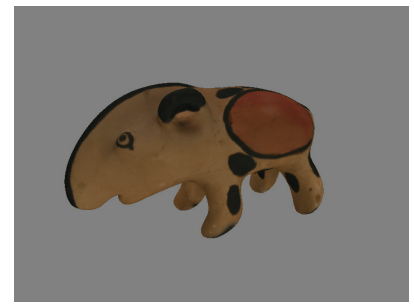

(a)

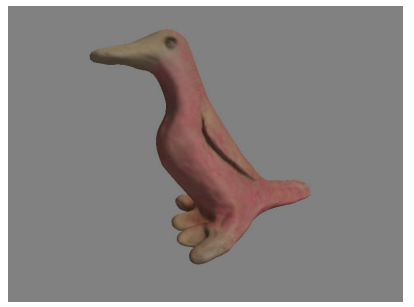

(b)
Fig. 3. 3D models of artifacts manufactured by two Brazilian indigenous communities: (a) Wauja and (b) Karaja.

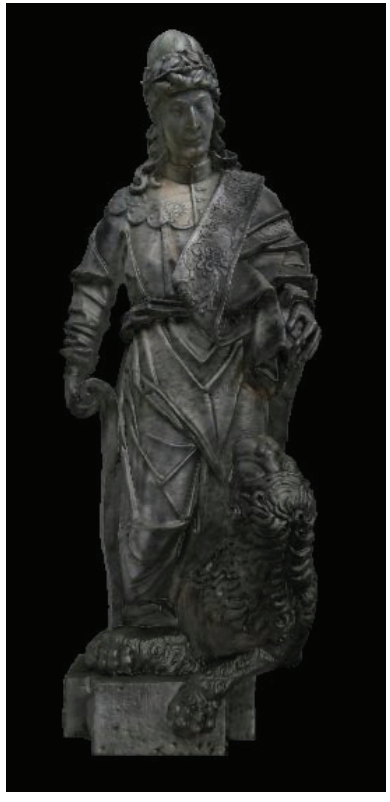

(a)

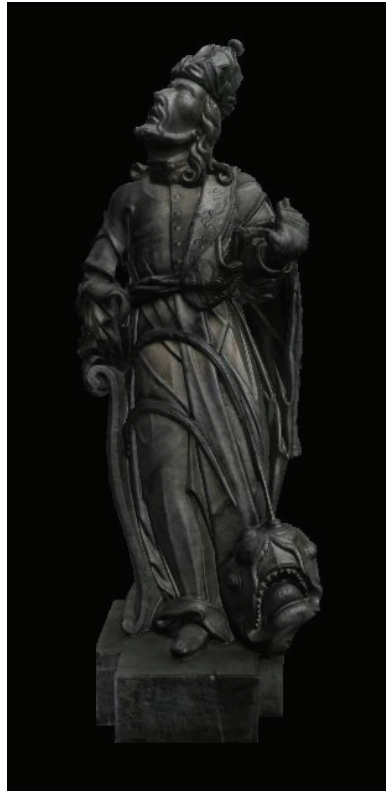

(b)
Fig. 4. Rendered 3D models of two preserved Prophets: (a) Prophet Daniel and (b) Prophet Jonas.

Currently we are improving our 3D reconstruction system by including other stages such as mesh simplification, progressive visualization of large 3D models and the estimation of the materials' appearance and reflectance properties.

\section{B. 3D Face Recognition}

The IMAGO Research Group has started developing a 3D face recognition system in 2005. Since then several works were published by our group presenting different components of a 3D face recognition system, such as face reconstruction [9], face localization [8], face segmentation [10], [15], facial landmarks detection [10], [15], face matching [11], [14], [16][18] and matching evaluation [12], [13].

This project emerged from the idea of using a registration technique proposed by our group, known as Surface Interpenetration Measure (SIM) [27], to evaluate the similarity between 
3D facial images. The SIM quantify the intersections between two surfaces, and registrations of facial images from the same person usually present more intersections than registrations of images from different subjects. An example of the registration process applied to facial images from the same person is shown in Figure 5.

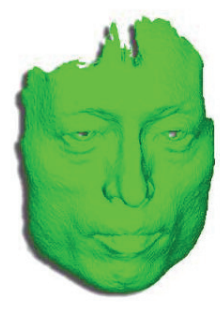

(a)

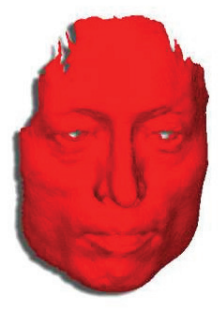

(b)

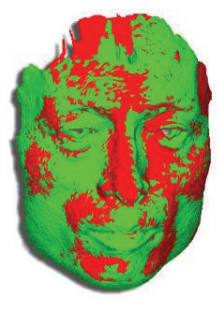

(c)
Fig. 5. Face registration for matching: (a),(b) input images and (c) the resulting registration.

Our initial works aimed to validate the SIM as a robust and reliable measure for face matching [17], [18]. The following works were focused on the development of a fully automatic 3D face recognition system. To this end, we created two new modules for face segmentation and facial landmark detection [10], [15]. The segmentation module finds and extracts the face of an input image and the landmark detection module locates some feature points to prealign facial surfaces. Some results of these approaches are shown in Figure 6.
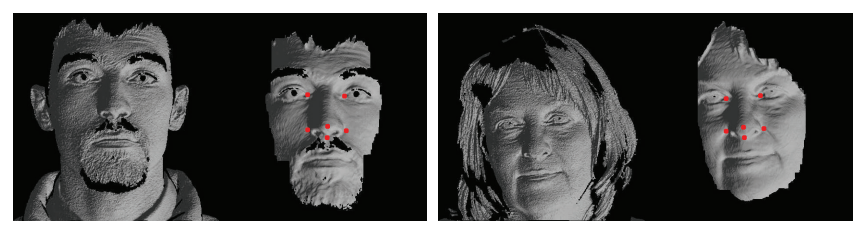

Fig. 6. Examples of face segmentation and landmarks detection processes.

The preprocessing modules were then extended in order to deal with facial expression variations, which is one of the biggest challenges for 3D face recognition. To this end, feature points were employed to extract some rigid regions of the face which are less affected by expressions, as shown in Figure 7. The matching of multiple facial regions is performed in hierarchical manner [11]-[14], [16] to obtain faster and more accurate face verification results.

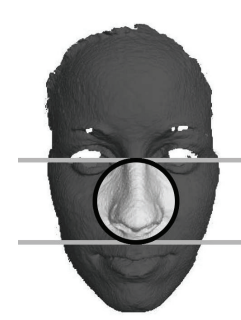

(a)

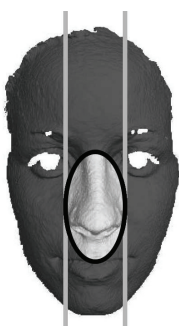

(b)

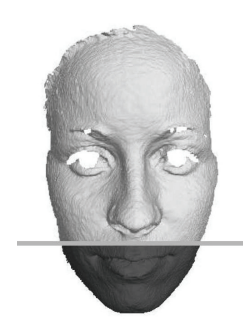

(c)
Fig. 7. Rigid regions extraction guided by the facial landmarks location.

Our group is working on 3D face recognition solutions in more difficult scenarios, such as real-time systems, recognition at distance and complex backgrounds. The initial works in this research line aim to locate faces in cluttered scenes [8] and to reconstruct the geometry of $2 \mathrm{D}$ face images to perform $3 \mathrm{D}$ face recognition at distance [9].

The current research on face recognition involves real-time 3D face matching, facial expression analysis and multimodal face recognition. This project has many applications in virtual reality and interactivity, such as user authentication, health applications (e.g. facial animation for treating autistic children), audiovisual applications (e.g. facial animation for videoconferences, games and multimedia), interactivity (e.g. using facial movements and expressions to perform commands) and realistic avatar modeling.

\section{FootScanAge and FootScanId}

The FootScanAge is a more accurate and automatic system to support gestational age determination [28]. To guarantee the newborn surveillance, specially premature newborns, it is extremely important to determine precisely the gestational age to guide diagnostics of the specialists in neonatology. In this context, the FootScanAge system presents a new alternative to manual methods, because it is non-invasive and estimates the gestational age by the computational analysis of the features extracted from the newborn plantar surface.

The system is composed by five main modules: the Image Acquisition and Search (IAS), the Image Processing Tool (IPT), the Data Mining Tool (DMT), Database (DB) and Web Interface (WI). The modules had been integrated into two basic tools: FootScanAgeDesktop (IAS, IPT and DMT) and FootScanAgeWeb (DB and WI). The IPT extracts features of the plantar surface of the newborns by applying different and interactive image processing techniques. The interface was developed with HCI requirements and allows handling, usability and user interaction during the image processing steps. The user can modify the parameters of the algorithms to obtain an improved image and guarantee more accuracy on the features extraction step by a supervised process. The DMT is based on machine learning algorithms and data mining models that utilize the features extracted on the IPT to estimate the final gestational age score (Figure 8).

Currently, the project also performs newborns identification (FootScanID) based on biometric characteristics (friction ridge patterns) of the newborns' palmprints. Newborn identification is crucial to avoid baby swaps in hospitals, child kidnapping and illegal adoptions. Despite the maturity of biometrics technology, there is no biometric system that has been developed for newborn identification purposes. Then, our efforts to develop a newborn recognition system [19] show that the automatic identification process is fully executable, although difficult. The ongoing stages of this project involves the development of a high resolution acquisition device, an enhanced acquisition protocol, the creation of a large database of newborn palmprints, and also the development of an interface allowing interactivity and usability to the user.

\section{Final Remarks}

The IMAGO Research Group has qualified more than 40 students and developed several solutions in its area of exper- 


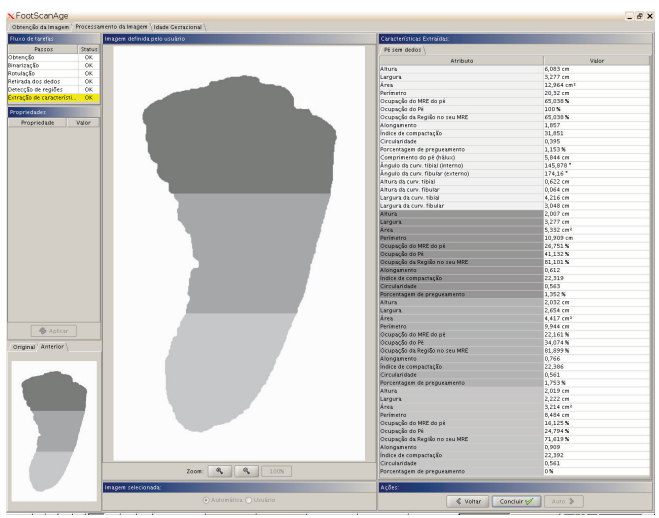

(a)

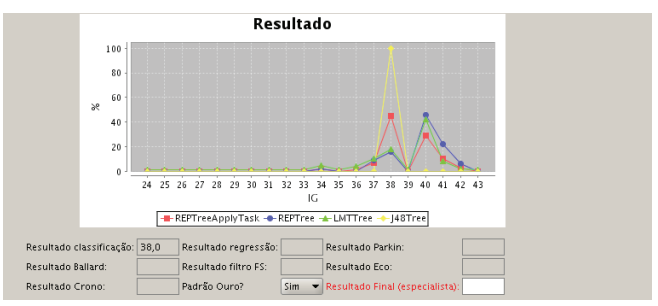

(b)

Fig. 8. FootScanAge: (a) feature extraction step on the IPT module and (b) the final result of the DMT module.

tise. It offers an active research environment which enables graduate and undergraduate students to participate in innovative projects with appropriate computing facilities, and also to interact with group members working on related projects. The students are also encouraged to attend conferences, symposia and workshops to expose the result of their works and actively engage with the scientific community. Details of how to join the group are available at the website.

\section{REFERENCES}

[1] L. Silva, O. R. P. Bellon, K. L. Boyer, and P. F. U. Gotardo, "Lowoverlap range image registration for archaeological applications," in Conference on Computer Vision and Pattern Recognition Workshop, 2003, pp. 9-9.

[2] L. Silva, O. R. P. Bellon, and K. L. Boyer, "Computer vision and graphics for heritage preservation and digital archaeology," Revista de Informática Teórica e Aplicada, vol. 11, no. 1, pp. 9-31, 2004.

[3] A. Vrubel, O. R. P. Bellon, and L. Silva, "A 3d reconstruction pipeline for digital preservation," in IEEE Computer Society Conference on Computer Vision and Pattern Recognition, 2009, pp. 2687-2694.

[4] J. O. Santos Junior, O. Bellon, L. Silva, and A. Vrubel, "Improving $3 \mathrm{~d}$ reconstruction for digital art preservation," in 16th International Conference on Image Analysis and Processing (Accepted), 2011.

[5] B. T. Andrade, C. M. Mendes, J. O. Santos Junior, O. R. P. Bellon, and L. Silva, "3d preserving xviii century barroque masterpiece: Challenges and results on the digital preservation of aleijadinho's sculpture of the prophet joel," Journal of Cultural Heritage (In Press), 2011.

[6] B. T. Andrade, O. R. P. Bellon, L. Silva, and A. Vrubel, "Digital preservation of brazilian indigenous artworks: Generating high quality textures for 3d models," Journal of Cultural Heritage (In Press), 2011.

[7] C. M. Mendes, D. R. Drees, L. Silva, and O. R. P. Bellon, "Interactive $3 \mathrm{~d}$ visualization of natural and cultural assets," in 2nd Workshop on eHeritage and digital art preservation, 2010, pp. 49-54.

[8] M. Pamplona Segundo, L. Silva, and O. R. P. Bellon, "Real-time scale-invariant face detection on range images," in IEEE International Conference on System, Man, and Cybernetics (Accepted), 2011.

[9] J. Choi, G. Medioni, Y. Lin, L. Silva, O. R. P. Bellon, M. Pamplona Segundo, and T. C. Faltemier, " $3 \mathrm{~d}$ face reconstruction using a single or multiple views," in 20th International Conference on Pattern Recognition, 2010, pp. 3959-3962.
[10] M. Pamplona Segundo, L. Silva, O. R. P. Bellon, and C. C. Queirolo, "Automatic face segmentation and facial landmark detection in range images," IEEE Transactions on Systems, Man, and Cybernetics, Part B: Cybernetics, vol. 40, no. 5, pp. 1319-1330, 2010.

[11] C. C. Queirolo, L. Silva, O. R. P. Bellon, and M. Pamplona Segundo, "3d face recognition using simulated annealing and the surface interpenetration measure," IEEE Transactions on Pattern Analysis and Machine Intelligence, vol. 32, no. 2, pp. 206-219, 2010.

[12] C. C. Queirolo, S. A. Drovetto Junior, L. Silva, O. R. P. Bellon, and M. Pamplona Segundo, "Hierarchical evaluation model: Extended analysis for $3 \mathrm{~d}$ face recognition," in Computer Vision and Computer Graphics. Theory and Applications, ser. Communications in Computer and Information Science, 2009, vol. 24, pp. 213-224.

[13] S. A. Drovetto Junior, L. Silva, and O. R. P. Bellon, "Hierarchical evaluation model for $3 \mathrm{~d}$ face recognition," in International Conference on Computer Vision Theory and Applications, 2008, pp. 67-74.

[14] C. C. Queirolo, L. Silva, O. R. P. Bellon, and M. Pamplona Segundo, " $3 \mathrm{~d}$ face recognition using the surface interpenetration measure: A comparative evaluation on the frgc database," in 19th International Conference on Pattern Recognition, 2008, pp. 1-5.

[15] M. Pamplona Segundo, C. C. Queirolo, O. R. P. Bellon, and L. Silva, "Automatic 3d facial segmentation and landmark detection," in 14th International Conference on Image Analysis and Processing, 2007, pp. 431-436.

[16] C. C. Queirolo, M. Pamplona Segundo, O. R. P. Bellon, and L. Silva, "Noise versus facial expression on $3 \mathrm{~d}$ face recognition," in 14th International Conference on Image Analysis and Processing, 2007, pp. $171-178$.

[17] O. R. P. Bellon, L. Silva, C. C. Queirolo, S. A. Drovetto Junior, and M. Pamplona Segundo, "3d face image registration for face matching guided by the surface interpenetration measure," in IEEE International Conference on Image Processing, 2006, pp. 2661-2664.

[18] O. R. P. Bellon, L. Silva, and C. C. Queirolo, "3d face matching using the surface interpenetration measure," in Image Analysis and Processing ICIAP 2005, ser. Lecture Notes in Computer Science, 2005, vol. 3617, pp. 1051-1058.

[19] R. Lemes, O. R. P. Bellon, L. Silva, and A. K. Jain, "Biometric recognition of newborns: Identification using palmprints," in International Joint Conference on Biometrics (Accepted), 2011.

[20] D. Weingaertner, O. R. P. Bellon, L. Silva, and M. N. L. Cat, "Newborn's biometric identification: Can it be done?" in International Conference on Computer Vision Theory and Applications, 2008, pp. 200-205.

[21] O. R. P. Bellon, M. N. L. Cat, L. Silva, and K. L. Boyer, "Using computer vision to help the determination of the gestational age of newborns," Academic Radiology, vol. 12, no. 5, pp. 544-553, 2005.

[22] O. R. P. Bellon, M. Severich, L. Silva, M. N. L. Cat, and K. L. Boyer, "Image analysis of newborn plantar surface for gestational age determination," in Medical Image Computing and Computer - Assisted Intervention, 2003, vol. 2879, pp. 472-479.

[23] L. Silva, O. R. P. Bellon, R. P. Lemes, J. A. Meira, and M. N. L. Cat, "An image processing tool to support gestational age determination," in Proceedings of the 19th IEEE Symposium on Computer-Based Medical Systems, 2006, pp. 867-874.

[24] L. Silva, O. R. P. Bellon, and P. F. U. Gotardo, "Mouselupe: An accessability tool for people with low vision," Usability: Gaining a Competitive Edge, pp. 191-202, 2002.

[25] O. R. P. Bellon and L. Silva, "New improvements to range image segmentation by edge detection," Signal Processing Letters, vol. 9, no. 2, pp. 43-45, 2002.

[26] P. F. U. Gotardo, O. R. P. Bellon, K. L. Boyer, and L. Silva, "Range image segmentation into planar and quadric surfaces using an improved robust estimator and genetic algorithm," IEEE Transactions on Systems, Man, and Cybernetics, Part B: Cybernetics, vol. 34, no. 6, pp. 23032316, 2004.

[27] L. Silva, O. R. P. Bellon, and K. L. Boyer, "Precision range image registration using a robust surface interpenetration measure and enhanced genetic algorithms," IEEE Transactions on Pattern Analysis and Machine Intelligence, vol. 27, no. 5, pp. 762-776, 2005.

[28] L. Silva, O. R. P. Bellon, R. P. Lemes, J. A. Meira, and M. N. L. Cat, "An image processing tool to support gestational age determination," in 19th IEEE Symposium on Computer-Based Medical Systems, 2006, pp. 867-874. 